\title{
The CMV early enhancer/chicken $\beta$ actin (CAG) promoter can be used to drive transgene expression during the differentiation of murine embryonic stem cells into vascular progenitors
} Annika N Alexopoulou ${ }^{1,2}$, John R Couchman ${ }^{1,3}$ and James R Whiteford ${ }^{* 1,2}$

Address: ${ }^{1}$ National Heart and Lung Institute, Sir Alexander Fleming Building, Faculty of Medicine, Imperial College London SW7 2AZ, UK, ${ }^{2} \mathrm{MRC}$ Prion Unit, Institute of Neurology, UCL, Queen Square, London WC1N 3BG, UK and ${ }^{3}$ Biomedicinsk Institute, University of Copenhagen, Biocenter, Ole Maoløes Vej 5, 2200 Copenhagen N, Denmark

Email: Annika N Alexopoulou - a.alexopoulou@prion.ucl.ac.uk; John R Couchman - john.couchman@bric.dk; James R Whiteford* - james.whiteford@bric.dk

* Corresponding author

Published: II January 2008

BMC Cell Biology 2008, 9:2 doi:10.1|86/I47|-2121-9-2
Received: II July 2007

Accepted: II January 2008

This article is available from: http://www.biomedcentral.com/l47I-2/2I/9/2

(C) 2008 Alexopoulou et al; licensee BioMed Central Ltd.

This is an Open Access article distributed under the terms of the Creative Commons Attribution License (http://creativecommons.org/licenses/by/2.0), which permits unrestricted use, distribution, and reproduction in any medium, provided the original work is properly cited.

\begin{abstract}
Background: Mouse embryonic stem cells cultured in vitro have the ability to differentiate into cells of the three germ layers as well as germ cells. The differentiation mimics early developmental events, including vasculogenesis and early angiogenesis and several differentiation systems are being used to identify factors that are important during the formation of the vascular system. Embryonic stem cells are difficult to transfect, while downregulation of promoter activity upon selection of stable transfectants has been reported, rendering the study of proteins by overexpression difficult.

Results: CCE mouse embryonic stem cells were differentiated on collagen type IV for 4-5 days, $\mathrm{FlkI}^{+}$mesodermal cells were sorted and replated either on collagen type IV in the presence of VEGFA to give rise to endothelial cells and smooth muscle cells or in collagen type I gels for the formation of vascular tubes. The activity of the CMV and $\beta$-actin promoters was downregulated during selection of stable transfectants and during differentiation to the FlkI stage, while the CMV immediate enhancer/ $\beta$-actin promoter in the PCAGIPuro-GFP vector led to $100 \%$ of stably transfected undifferentiated and differentiated cells expressing GFP. To further test this system we expressed syndecan- 2 and -4 in these cells and demonstrated high levels of transgene expression in both undifferentiated cells and cells differentiated to the FlkI stage.

Conclusion: Vectors containing the CAG promoter offer a valuable tool for the long term expression of transgenes during stem cell differentiation towards mesoderm, while the $\mathrm{CMV}$ and $\beta$-actin promoters lead to very poor transgene expression during this process.
\end{abstract}

\section{Background}

Mouse embryonic stem (ES) cells are derived from the inner cell mass (ICM) of the mouse blastocyst and retain pluripotency when cultured in vitro in the presence of factors that inhibit differentiation, such as leukaemia inhibitory factor (LIF). However, when these factors are withdrawn, ES cells have the potential to differentiate into ectodermal, mesodermal, endodermal and germ cell lineages (reviewed in [1]). ES cells can differentiate either in suspension, forming embryoid bodies (EBs) or as twodimensional adhesion cultures, either in the presence of feeder cells or extracellular matrix (ECM) molecules [1]. 
The regulation of ES cell differentiation is currently under intense study, not least for potential therapeutic use. One important area of investigation is ES cell differentiation either in EBs or in 2-dimensional culture, along pathways recapitulating vasculogenesis and early angiogenesis $[2,3]$. Related to this is delineation of functions for growth factors, adhesion molecules and transcription factors in the formation of the vasculature.

Elucidating the role of angiogenic factors during differentiation often requires over-expression of wild type and mutant proteins. Transfection of ES cells with plasmids using traditional electroporation and lipofectaminebased methods can be problematic [4-6]. Conflicting data about the activity of different promoters in undifferentiated ES cells have also been obtained, while several studies have suggested that transcriptional inactivation of promoters occurs during selection of stably transfected cells [7-9]. Another problem is obtaining stable transgene expression through stem cell differentiation. Several studies have looked at the efficacy of promoters in plasmid based vector systems with a view to obtaining high transient and stable expression in stem cells. For example, the CAG promoter has been used to express transgenes in undifferentiated ES cells as well as ES cells undergoing differentiation towards neuronal, myogenic and mesodermal cell types [10-12]. In the latter case a transfection system was used (ES cells expressing polyoma large T antigen and a vector with a polyoma virus origin of replication) leading to episomal propagation of the vector carrying the transgene of interest [12]. Although episomal plasmid propagation may lead to very high levels of transgene expression due to a high copy number of plasmid per cell, certain experiments may require lower levels of overexpression that may be more physiologically relevant. Here, we compare the promoter activity of the CMV, chicken $\beta$-actin and CAG promoters in undifferentiated murine embryonic CCE cells as well as during differentiation to mesoderm. These cells are routinely used for differentiation into mesodermal lineages [13] and do not express the polyoma virus large $\mathrm{T}$ antigen allowing integration of transgenes into the host's genome. We demonstrate that of these vector systems, only plasmids containing the CAG promoter can be used for long term expression of genes during differentiation of two dimensional cultures of mouse embryonic CCE ES cells grown on collagen substrates towards mesoderm.

\section{Results and Discussion Differentiation of mesodermal cell lineages from mouse CCE ES cells}

Cells of a mesodermal/endothelial lineage were differentiated from mouse CCE embryonic stem cells following the protocol described in [13] (Fig. 1A). Undifferentiated CCE cells expressed high levels of E-cadherin, SSEA-1 and
Pecam-1, which is consistent with previous data [14-16]. These cells did not express the mesodermal/endothelial marker Flk1, the endothelial cell (EC) marker VE-cadherin or the smooth muscle cell (SMC)/pericyte marker PDGFR $\beta$ (Fig. 1B, day 0). Differentiation was initiated by the removal of LIF from the medium and the seeding of cells on collagen type IV. Flk1 expression was observed on days 4-5 of differentiation, while elevated levels of PDGFR $\beta$ were also noted at the same time. Expression of Pecam-1 was much reduced during differentiation and the levels of E-cadherin and SSEA-1 also declined by day 5 . VE-cadherin levels remained negligible during this 5 day differentiation period (Fig. 1B).

Flk1+ cells from either day 4 or day 5 cultures were FACS sorted and replated on collagen type IV-coated plates in the presence of VEGFA for a further 3 days (Fig. 1A). Double immunofluorescence microscopy for the EC markers VE-cadherin or Pecam-1 and the SMC marker $\alpha$-smooth muscle actin ( $\alpha$ SMA) (Fig. 1C, D) showed the presence of EC sheets surrounded by SMCs. Differentiation into ECs was VEGFA-dependent, as only $\alpha \mathrm{SMA}^{+}$cells were observed in its absence (data not shown). When Flk $1^{+}$cells were FACS sorted and plated in three dimensional collagen type I gels in the presence of VEGFA for an additional 6 days, the formation of angiogenic sprouts was observed (Fig. 1E, F). Tubular structures positive for the EC marker Pecam- 1 were surrounded by $\alpha \mathrm{SMA}^{+} \mathrm{SMCs} /$ pericytes (Fig. $1 G, H)$. The formation of tubes was dependent on VEGFA/Flk1 signalling, since no tubes formed in the absence of VEGFA or when Flk1- cells were sorted and cultured in the presence of VEGFA (data not shown). These data are in agreement with previous studies $[13,3]$ and suggest that this differentiation protocol mimics aspects of both vasculogenesis and early angiogenesis and is suitable for ectopic expression studies.

\section{The CMV and $\beta$-actin promoters are not suitable for sustained expression of transgenes during CCE cell differentiation}

High levels of transfection efficiency $(80 \%+)$ of CCE cells (Fig. 2A, B) were obtained with a modified lipofectamine based protocol where suspended cells were transfected with pEGFP-N1 [9]. This compared favourably with other methods such as electroporation $(70 \%$ transfection efficiency; data not shown) and the standard lipofectamine protocol ( $40 \%$ efficiency; data not shown). However the longevity of GFP expression in transfected CCE cells was poor. After 7 days in culture under antibiotic selection very few fluorescing cells were evident (Fig. 2C, D) as compared to cultures 2 days after transfection (Fig. 2A, B). These results were confirmed by FACS analysis showing that only $34-50 \%$ of undifferentiated cells surviving antibiotic selection expressed GFP (Fig. 2G, I), while a dramatic decrease in the transgene's expression was noted in 


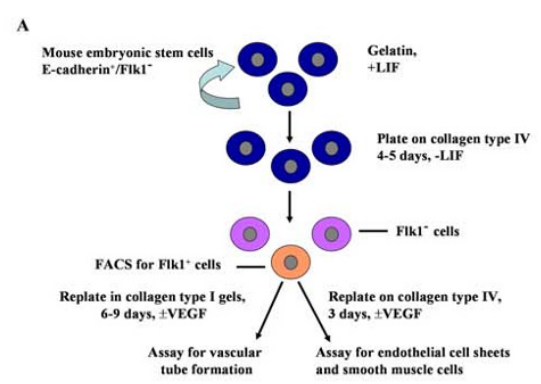

B

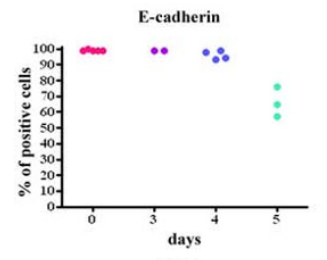

Flk1

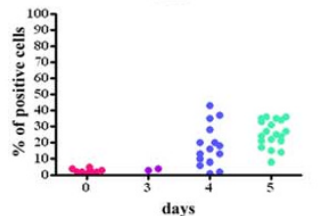

days

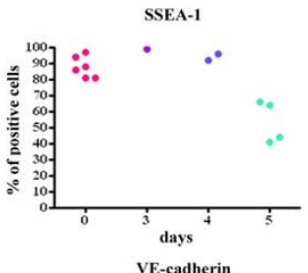

VE-cadherin

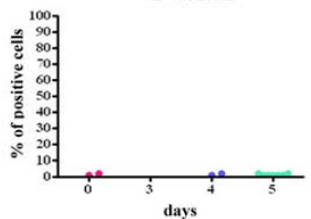

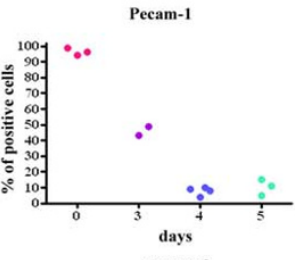

PDGFRB

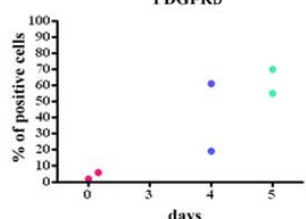

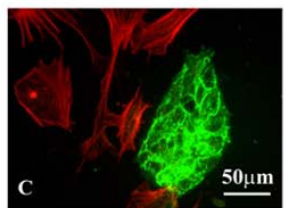
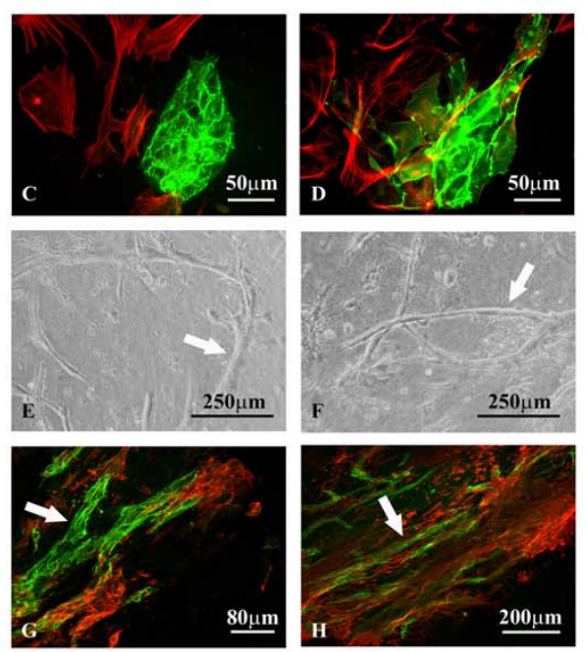

\section{Figure I}

ES cell differentiation into Flk $\mathbf{I}^{+}$mesodermal, endothelial and smooth muscle cells. A. Schematic showing the protocol for differentiation of CCE cells to mesodermal lineages. B. Expression of cell surface markers at day 0 and days 3-5 of differentiation; cells were analysed by FACS for the markers shown. C-D. FlkI ${ }^{+}$cells were plated on collagen type IV in the presence of VEGFA. Cells were double immunostained for VE-cadherin (green) and $\alpha$ SMA (red) (C) or PECAM-I (green) and aSMA (red) (D). EC sheets were found surrounded by SMCs. E-F. Phase contrast micrographs of Flk I+ cells after FACS sorting and seeding into collagen type I gels in the presence of VEGFA for 6 days. Vascular tube structures are arrowed. G-H. Collagen I gels were sectioned and stained for Pecam-I (green) and $\alpha$ SMA (red) and showed Pecam- I+ tubular structures (arrows) surrounded by smooth muscle cells. 
stably transfected cells differentiated for 5 days on collagen type IV to the Flk1 stage (Fig. 2H, I). It should be noted that at this stage the pool of cells contain both Flk1+ and Flk1- cells. The GFP cDNA in pEGFP-N1 is under the control of the CMV promoter and it is not uncommon for transcriptional inactivation of this promoter to occur in certain cell types. To overcome this problem we expressed GFP under the control of the chicken $\beta$-actin promoter. GFP expression in differentiated cells was again very low (Fig. 2I) and this promoter offered no advantage over the CMV promoter.

Since a higher percentage of transiently transfected cells expressed GFP compared to the stably transfected cells (Fig. 2A-D), cells 2 days post transfection were used for differentiation to test whether this would lead to more $\mathrm{GFP}^{+}$cells present at the Flk1 stage. However, the use of transiently transfected cells resulted in no cells expressing
GFP on day 5 of differentiation (Fig. 2E, F). This suggested that stable integration of the transgene into the host's chromosome is required for its expression.

From the above studies it was evident that resistance against antibiotic selection did not correlate with expression of the transgene of interest. Therefore, bicistronic vectors having GFP as the reporter gene were tested, as they would allow for easy identification and selection of cells expressing the transgene of interest. CCE cells were transfected with either the pIRES2-EGFP plasmid or pIRES2$\operatorname{EGFP}(\beta$-actin $)$, where the CMV promoter had been substituted for the chicken $\beta$-actin promoter. However, GFP expression was not apparent in CCE cells transfected with either plasmid even 1 day after transfection (Figure 3A). Control cultures were transfected in parallel with pEGFP$\mathrm{N} 1$ and $\mathrm{GFP}^{+}$cells were abundant in this case. The bicistronic vectors were then transfected into fibroblasts to
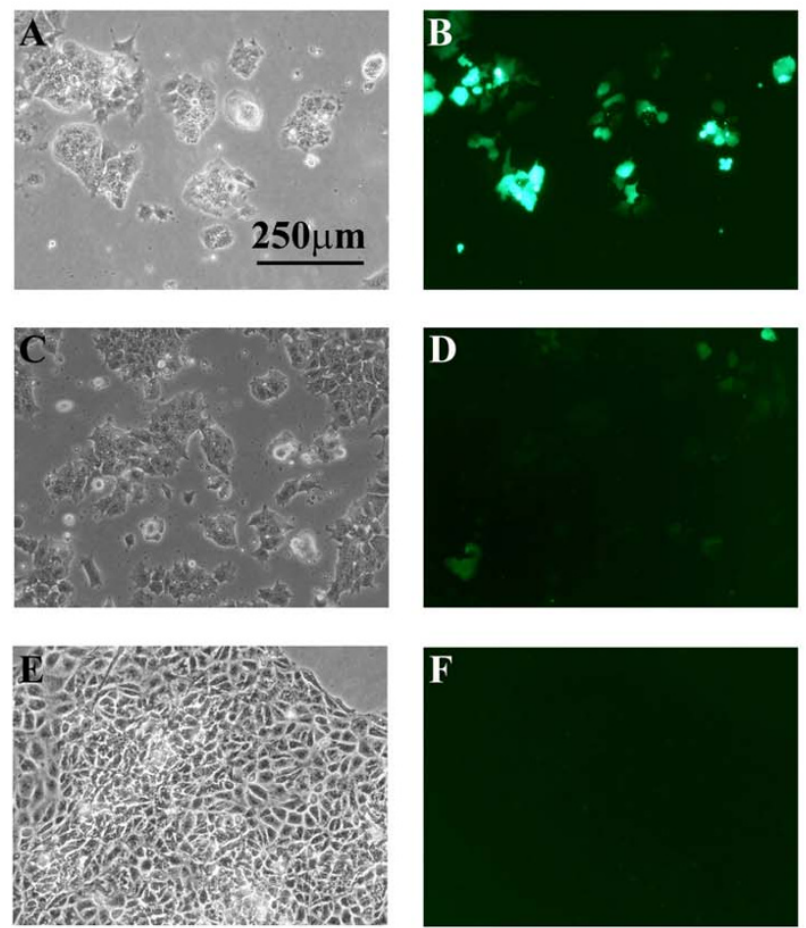

G

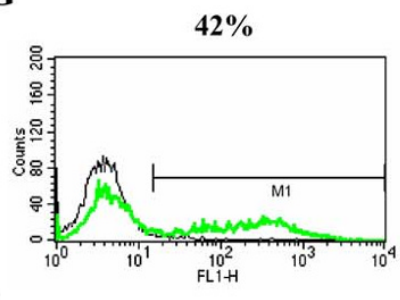

H

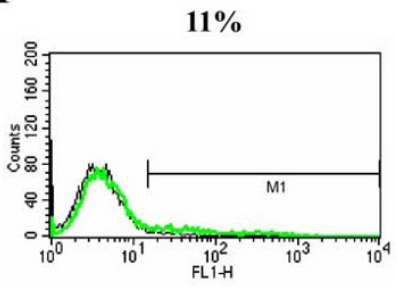

\begin{tabular}{|l|l|l|}
\hline Cells & Vector & $\%$ of GFP ${ }^{+}$cells \\
\hline Undifferentiated & pEGFP-N1 & $34-50$ \\
\hline Differentiated to the Flk1 stage & pEGFP-N1 & $10-15$ \\
\hline Differentiated to the Flk1 stage & pLK444-GFP & 19 \\
\hline
\end{tabular}

\section{Figure 2}

The CMV promoter is down regulated in mouse embryonic CCE cells and during differentiation into mesoderm. A-D. CCE cells were transfected with pEGFP-NI and subjected to G4I 8 selection 48 hours later. Cells expressing GFP are obvious 2 days after transfection (A, B) while few GFP positive cells remain 7 days post transfection (C, D). E-F. CCE cells were transfected with pEGFP-NI and differentiation was initiated 2 days post transfection. No GFP cells were observed on day 5 of differentiation to the FlkI stage (pool of containing both Flk I+ and Flk I- cells) when transient transfectants were used. G-I. CCE cells were transfected with PEGFP-NI and placed under G4I8 selection 2 days post transfection. Undifferentiated cells $(\mathrm{G}, \mathrm{I})$ and cells differentiated to the FlkI stage $(\mathrm{H}, \mathrm{I})$ were analysed by FACS for GFP expression I4-I9 days post transfection. The data suggest that less than $50 \%$ of the undifferentiated cells express GFP, while this percentage declines during differentiation to the FlkI stage. Similar levels of GFP expression were obtained during differentiation of cells transfected with pLK444-GFP. 
check their functionality and fluorescing cells were obtained from transfections with both pIRES2-EGFP and pIRES2-EGFP( $\beta$-actin) (Fig. 3B).

The above data suggest that to obtain sustained transgene expression in murine CCE cells and during differentiation into mesoderm, alternative promoters to the CMV and the chicken $\beta$-actin promoters are required. Furthermore, the above promoters driving expression in a vector that contains an IRES element were inactive in undifferentiated ES cells, in agreement with previous reports [7].

\section{Vectors containing the CAG promoter can be used to express transgenes in murine CCE cells and during differentiation into mesoderm}

The CMV immediate enhancer/ $\beta$-actin (CAG) promoter has been shown to give higher levels of transgene expression in several cell lines compared to the CMV and $\beta$-actin promoters [17]. Two different vectors containing the CAG promoter were used. The first, pCAGIPuro is a bicistronic

\section{CCE}

A
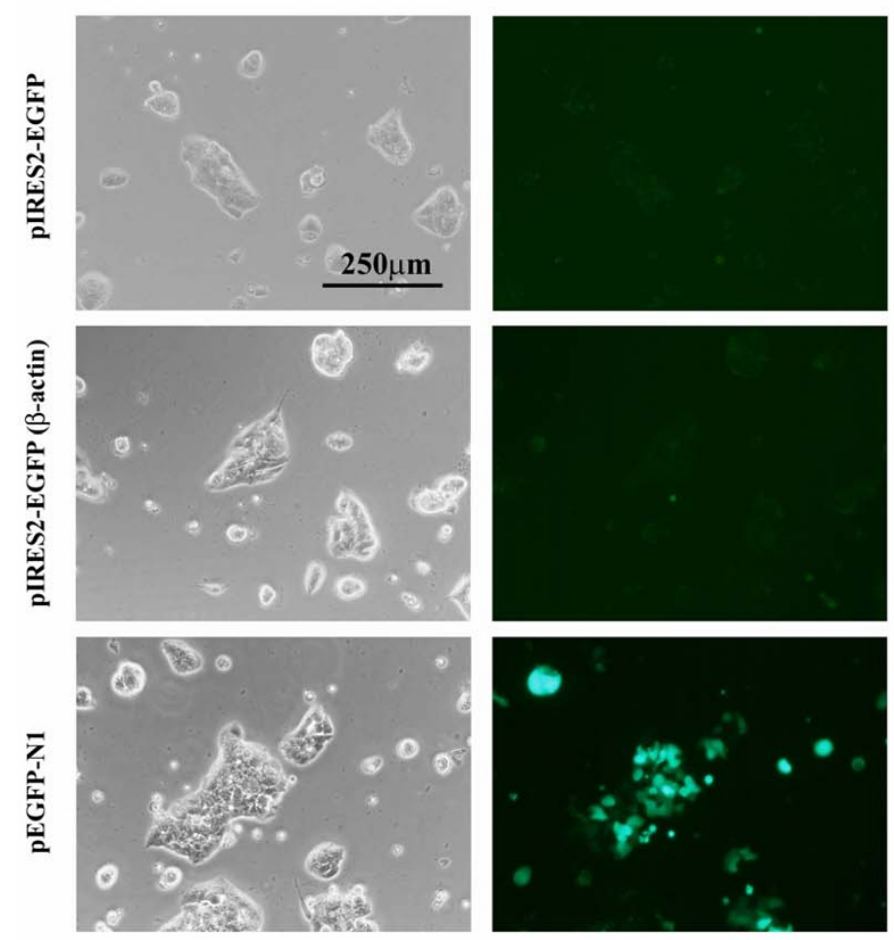

vector, where a puromycin resistance gene follows the IRES element. GFP was cloned into this vector to create pCAGIPuro-GFP (Table 1). The pCAGGS plasmid is a monocistronic vector with GFP expression also under the control of the CAG promoter. The G418 resistance cassette from vector pMC1neo polyA was cloned into pCAGGS to create pCAGGSneo-GFP (Table 1).

CCE cells were transfected with pCAGIPuro-GFP and selected for puromycin resistance. FACS analysis for GFP expression showed that virtually $100 \%$ of either undifferentiated (Fig. 4A) or cells differentiated to the Flk1 stage (Fig. 4B) expressed high levels of GFP. Indeed, all of the cells in these differentiated cultures consisting of both Flk1+ and Flk1- cells were expressing GFP and this suggests that the CAG promoter is active in both Flk1+ and Flk1- cells. CCE cells were also transfected with the pCAGGSneo-GFP vector and were selected for G418 resistance. When stable transfectants were analysed by FACS, approximately 50\% of either undifferentiated (Fig.

\section{Figure 3}

The CMV and chicken $\beta$-actin promoters cannot drive gene expression in bicistronic vectors transfected into CCE cells. CCE cells $(A)$ or rat embryo fibroblasts $(B)$ were transfected with the bicistronic vectors pIRES2-EGFP and pIRES$\operatorname{EGFP}(\beta$-actin) in which the CMV promoter had been substituted with the chicken $\beta$-actin promoter. GFP expression was not evident in CCE cells transfected with these vectors; GFP+ cells were only obtained when cells were transfected with $p E G F P$ NI. In contrast GFP expression was apparent in fibroblasts transfected with all three constructs (B). Micrographs were obtained 24 hours after transfection. 
Table I: Plasmids used in this study.

\begin{tabular}{lcccc}
\hline Plasmid & Promoter & Transgene/s & IRES element & Antibiotic resistance to \\
\hline PEGFP-NI & CMV & GFP & No & G4I8 \\
PIRES2-EGFP & CMV & GFP & Yes & G4I8 \\
PIRES2( $\beta$-actin) & $\beta$-actin & GFP & Yes & G4I8 \\
PLK444-GFP & $\beta$-actin & GFP & No & G4I8 \\
PCAGIPuro-GFP & CAG & GFP and Puromycin resistance & Yes & Puromycin \\
PCAGGSneo-GFP & CAG & GFP & No & G4I8 \\
PCAGIPuro-sdc2/4 & CAG & Syndecan-2/4 & Yes & Puromycin \\
\hline
\end{tabular}

4C) or differentiated (Fig. 4D) cells were GFP+. While this was a substantially lower percentage of positive cells than that obtained with the pCAGIPuro-GFP vector, it was much superior to the pEGFP-N1 and pLK444-GFP vectors, particularly during differentiation (see Fig. 2I).
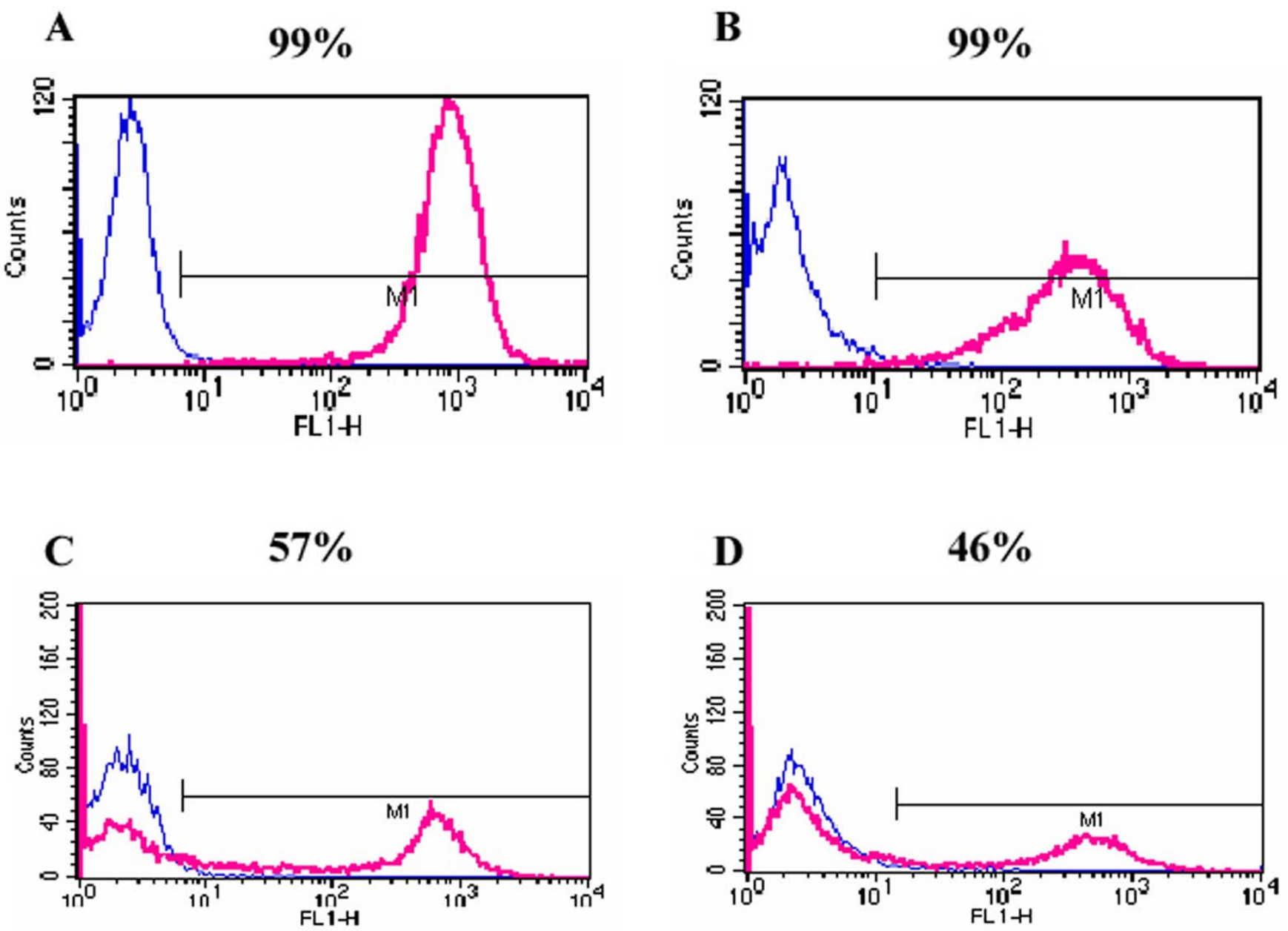

\section{Figure 4}

Sustained GFP expression in CCE cells during differentiation into mesoderm is obtained using the CAG promoter. CCE cells were transfected with PCAGIPuro-GFP (A, B) or PCAGGSneo-GFP (C, D) and selected for puromycin or G4I 8 resistance, respectively. FACS analysis was performed on undifferentiated CCE cells (A, C) and cells differentiated to the Flkl stage $(B, D) \mid 4-18$ days posts transfection and GFP expression was compared in vectors expressing GFP (red) and empty vector (blue) transfected controls. 
Having shown that the pCAGIPuro vector could be used to achieve sustained GFP expression during differentiation of CCE cells to mesodermal cell types we tested this vector system for its ability to express genes of biological interest. The syndecans are type I transmembrane proteoglycans involved in cell adhesion, migration and growth factor interactions [18]. In mammals there are four family members and we analysed undifferentiated and differentiating CCE cells for syndecan expression using RT-PCR. Undifferentiated CCE cells expressed only syndecans-1 and -4 (Fig. 5A), while Flk $1^{+}$and Flk1- cells FACS sorted on day 4 of differentiation expressed all four syndecans (Fig. $5 \mathrm{~B}, \mathrm{C})$. We then decided to study syndecans- 2 and -4 in further detail as they have been implicated in playing a role in the vascular system [19-23]. FACS analysis of undifferentiated CCE cells confirmed that cell surface syndecan-4 is present in these cells whereas syndecan- 2 is not (Fig. 5D). It was only after differentiation that syndecan-2 appeared on the cell surface at low levels.

We then used the pCAGIPuro plasmid to express syndecans- 2 and - 4 . Transfected cells were selected for puromycin resistance and the cells were differentiated on collagen type IV for 5 days. FACS analysis confirmed that high levels of cell surface expression of both proteins was achieved in undifferentiated as well as differentiated cells (Fig. 5D), although overexpression of these receptors had no effect on the percentage of Flk1+ cells obtained during differentiation (Fig. 5E).

\section{Conclusion}

Here we optimised a differentiation system of ES cells into Flk1+ mesodermal cells, ECs and SMCs and subsequently used the cells to form angiogenic sprouts in vitro. We then evaluated a number of plasmid vector systems for expressing transgenes during differentiation to Flk1+mesodermal cells and found that vectors which utilise the CAG promoter are most effective at driving gene expression and do not compromise the ability of stem cells to differentiate. The CAG promoter provides long term expression of transgenes in these cells however the system is most effective when the cells can be put under a selective pressure to express the transgene. For this reason bicistronic vectors containing an antibiotic resistance gene offers the best way of obtaining expression of transgenes during differentiation into mesodermal Flk1+ cells. This is in contrast to vectors containing the $\mathrm{CMV}$ and $\beta$-actin promoters which gave very low levels of expression. This presents a very useful tool for use in studies of angiogenesis and vasculogenesis.

\section{Methods}

\section{Cell lines and culture conditions}

CCE mES cells (StemCell Technologies) were grown in flasks coated with $0.1 \%$ gelatin (StemCell Technologies) in Dulbecco's Modified Eagle Medium (DMEM) (StemCell Technologies) supplemented with $15 \%$ foetal calf serum (FCS) (StemCell Technologies), $100 \mu \mathrm{M}$ 2-mercaptoethanol (Invitrogen), $10 \mathrm{ng} / \mathrm{ml}$ LIF (StemCell Technologies), $2 \mathrm{mM}$ glutamine (Invitrogen) and $100 \mu \mathrm{M}$ MEM non-essential amino acids (StemCell Technologies). The CCE cells were maintained in a humidified incubator at $37^{\circ} \mathrm{C}$ and $5 \% \mathrm{CO}_{2}$ in air. Rat embryo fibroblasts (REFs) were cultured in alpha-Minimum Essential Medium ( $\alpha M E M$; Cambrex) supplemented with 5\% FCS (Cambrex).

\section{Antibodies}

The Flk1 (clone Avas $12 \alpha$; final concentration $50 \mu \mathrm{g} / \mathrm{ml}$ ), the Pecam-1 (clone MEC13.3; $5 \mu \mathrm{g} / \mathrm{ml}$ ) and the VE-cadherin (clone 11D4.1; $50 \mu \mathrm{g} / \mathrm{ml}$ ) antibodies were from BD Pharmingen. The $\alpha$ smooth muscle actin ( $\alpha \mathrm{SMA})$ antibody $(1 \mathrm{~A} 4 ; 1 \mu \mathrm{g} / \mathrm{ml})$ was from Sigma, the E-cadherin antibody (clone ECCD2; $20 \mu \mathrm{g} / \mathrm{ml}$ ) was from Takara, Japan and the PDGFR $\beta$ antibody $(10 \mu \mathrm{g} / \mathrm{ml})$ was a kind gift from Dr W. Stallcup, Burnham Institute, La Jolla, California. R-Phycoerythrin-labelled SSEA-1 (clone 480; $4 \mu \mathrm{g} /$ $\mathrm{ml}$ ) and syndecan-2 antibodies (clone M140; $10 \mu \mathrm{g} / \mathrm{ml}$ ) were from Santa Cruz. The polyclonal chicken anti-syndecan-4 antibody (Harlan Sera-Lab, UK) was raised against the N-terminal 20 amino acids of the mature human protein. Antibodies were affinity purified from plasma using standard procedures. The secondary antibodies (Molecular Probes) used were: Alexa-488 labelled donkey anti-rat IgG; Alexa-568 labelled goat anti-mouse IgG; Alexa-488 labelled goat anti-rabbit IgG; Alexa-488 labelled goat antichicken IgY, with working concentrations of $10 \mu \mathrm{g} / \mathrm{ml}$.

\section{Flow cytometry analysis and sorting (FACS)}

Cells were detached with cell dissociation buffer (Invitrogen) and stained in FACS buffer (0.5\% BSA, 2 mM EDTA in $1 \times$ PBS) at $4^{\circ} \mathrm{C}$. For exclusion of dead cells, the nucleic acid dye Topro-3 (0.1 $\mu \mathrm{M}$; Molecular Probes) was used. The cells were analysed by a four colour FACS Calibur (BD) and data for 10,000 live cells were analysed by the CellQuest software. Cells stained with secondary antibody only or empty vector transfected cells served as controls for background fluorescence. The gate M1 was set to include $1 \%$ of the control cells that exhibited the highest fluorescence within the population and cells were considered to be positive when contained within the gate M1. For FACS sorting, dead cells were excluded by propidium iodide staining $(0.5 \mu \mathrm{g} / \mathrm{ml}$; Molecular Probes). Cell populations were sorted by FACS Diva (BD) and collected in sterile tubes in $2 \mathrm{ml}$ medium. Flk1+ cells were plated either on collagen type IV-coated plates or in collagen type I gels.

\section{Immunofluorescence microscopy}

Cells on glass coverslips were fixed in acetone $\left(-20^{\circ} \mathrm{C}\right)$ for 2 min. After washing in PBS, cultures were incubated with 

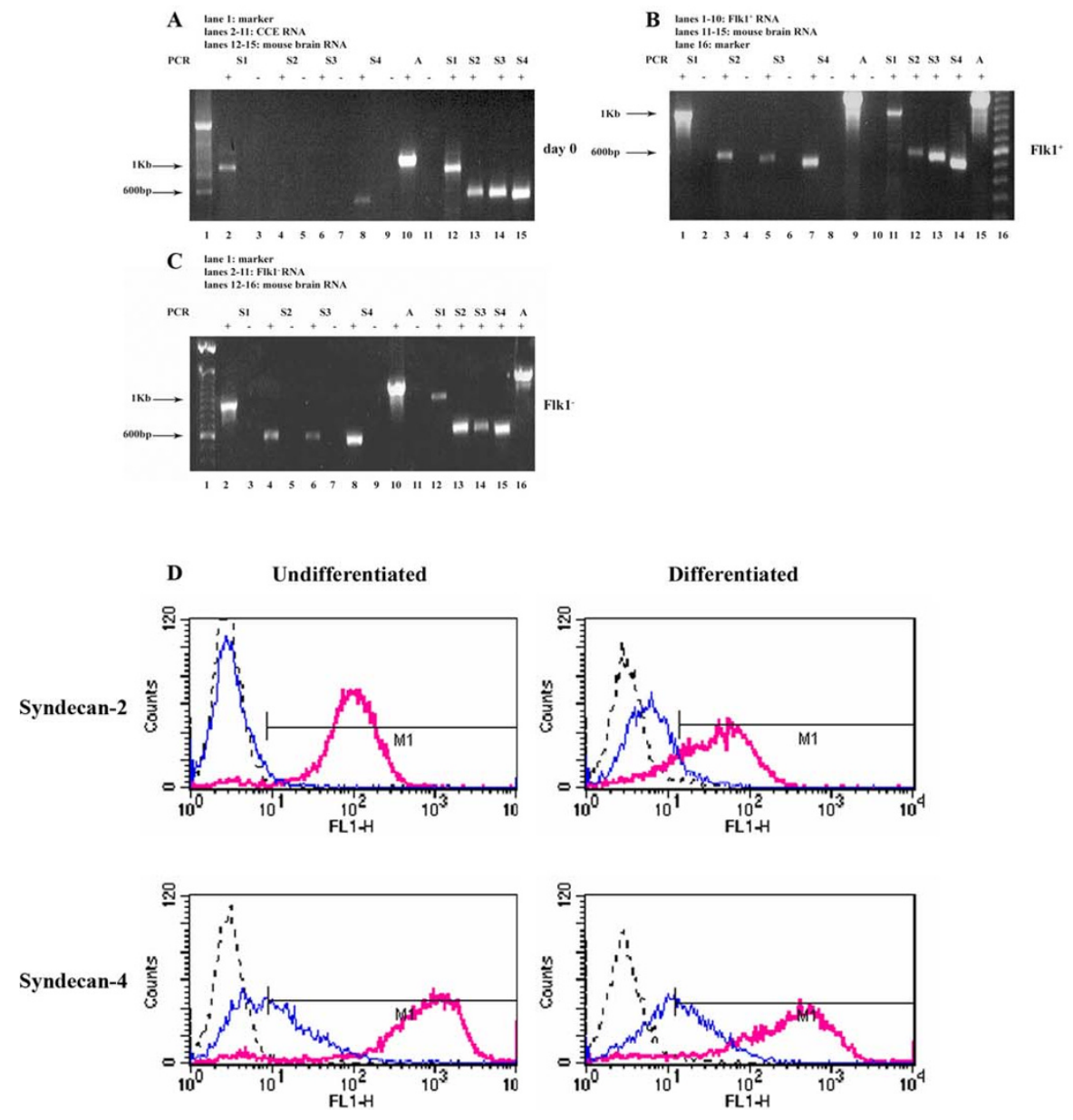

$\mathbf{E}$

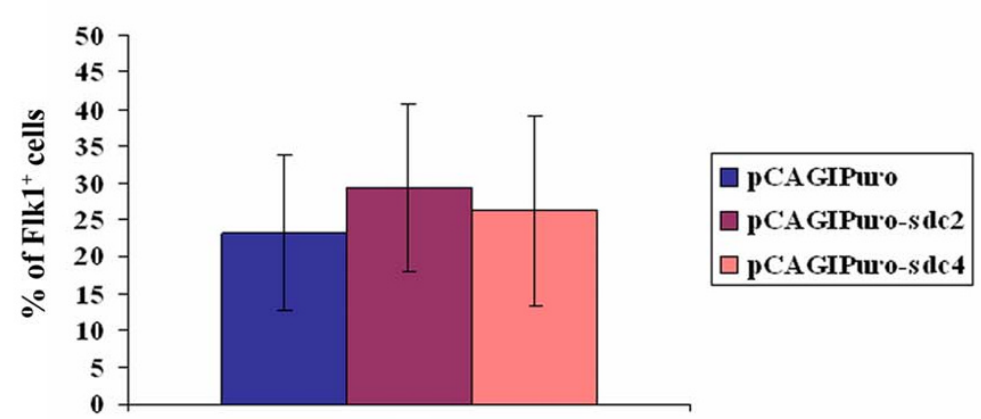

Figure 5

Ectopic expression of Syndecan-2 and -4 through differentiation of CCE cells to mesodermal lineages. Undifferentiated CCE cells (A) as well as FlkI+ $(B)$ and Flk - (C) cells FACS sorted on day 4 of differentiation were analysed by RT-PCR for syndecan expression. D. CCE cells were transfected with either PCAGIPuro-sdc2 or pCAGIPuro-sdc4 and were selected for puromycin resistance. FACS analysis was performed on undifferentiated and cells differentiated to the FlkI stage as indicated using antibodies against syndecan-2 and -4 . In each analysis, the dotted black line denotes the relevant secondary antibody control, the blue line corresponds to cells transfected with PCAGIPuro empty vector and the red line are cells transfected with constructs containing syndecan-2 or-4 cDNA. E. Levels of FlkI were measured on day 5 of differentiation in cells transfected with PCAGIPuro-sdc2, PCAGIPuro-sdc4 or empty vector control. Data from 5 independent experiments are shown, where a total of 9 flasks were analysed for each construct. The bars represent the standard deviation, and the results were analysed with repeated-measures ANNOVA, using Prism Graphpad 4.0, assuming Gaussian distribution. No statistical significance was observed for the difference in FLKI expression in cells transfected with the different constructs. 
the appropriate concentration of primary antibody in PBS for $1 \mathrm{~h}$ at $37^{\circ} \mathrm{C}$. Secondary antibody incubation for $1 \mathrm{~h}$ at $37^{\circ} \mathrm{C}$ was followed by further washing in PBS and coverslips were mounted with Immuno-Fluor (MP Biomedicals). Controls included staining with secondary antibody only, while in double labelling experiments, controls included incubation with one primary antibody and both secondary antibodies. Inappropriate cross-reactivity was minimal. Samples were examined on an Olympus Provis AX module fluorescence microscope (objective: UPlanApo $40 \times 1.0$ oil). The images were collected using a SPOT Insight Mono digital camera and were processed by Adobe Photoshop 7.0.

\section{ES cell differentiation into mesodermal, endothelial and smooth muscle cells}

Differentiation of CCE cells first into Flk $1^{+}$mesodermal cells and then into VE-cadherin ${ }^{+}$ECs and $\alpha \mathrm{SMA}^{+}$SMCs that were able to form angiogenic sprouts was achieved following the protocol described in [13]. Micrographs were taken on an Olympus DP50 microscope and digital camera system. Images were processed using the Viewfinder Lite software and Adobe Photoshop 7.0.

Three dimensional collagen type I cultures were fixed with $3.5 \%$ paraformaldehyde for $1 \mathrm{~h}$ at room temperature. The samples were washed $3 \times 15$ min with PBS and were released from the wells and embedded in an $8 \%$ gelatin solution to provide rigidity. Fixation with paraformaldehyde was repeated for $1 \mathrm{~h}$ at room temperature. Excess gelatin was removed and samples were stored in $0.02 \%$ Sodium Azide in $1 \times$ PBS at $4{ }^{\circ} \mathrm{C}$. Sections of $200 \mu \mathrm{m}$ thickness were prepared on a Micro-Cut H1200 vibrotome (Bio-Rad). Sections were permeabilised with $0.1 \%$ Triton
$\mathrm{X}-100$ for $1 \mathrm{~h}$ at room temperature, then washed $3 \times 15$ min with PBS. Primary antibodies against Pecam- 1 and aSMA were added at appropriate concentrations and incubated overnight at $4^{\circ} \mathrm{C}$. Sections were washed with PBS, then incubated in secondary antibody for $5 \mathrm{~h}$ at room temperature. After further extensive washing, sections were mounted onto slides using Gene Frame gaskets (Advanced Biotechnologies) and were sealed with coverslips. The samples were examined on a Leica DM-IRBE confocal microscope and images were processed using Leica TCS-NT and Adobe Photoshop 7.0 software.

\section{RNA extraction and RT-PCR}

RNA extraction was performed with RNA-Bee (Tel-test, inc., Texas, US) and reverse transcription was performed using Superscript II (Invitrogen) according to the manufacturer's instructions. OligodT was used to initiate cDNA synthesis and the syndecan gene specific primers are as detailed in Table 2.

\section{Vectors and Cloning}

The pEGFP-N1 plasmid was from Clontech. The pMC1neo PolyA plasmid was from Stratagene. The pCAGGS plasmid was a kind gift from Dr C. Huxley [17], Imperial College London. The pCAGIPuro vector was a kind gift from Dr H. Niwa, Riken Centre for Developmental Biology, Japan (Table 1).

Full length syndecan-2 and -4 cDNA obtained from RTPCR of mouse brain was cloned into the EcoRI site of vector pCAGIPuro to create pCAGIPuro-sdc2 and pCAGIPuro-sdc4, respectively using the primers described in Table 2.

Table 2: Primers used in this study.

\begin{tabular}{lll}
\hline Primer & Sequence & Purpose \\
\hline SDCIfor & ATGAGACGCGCGGCGCTC & RTPCR \\
SDCIrev & GGCGTAGAACTCCTCCTGC & RTPCR \\
SDC2for & GCCTTGATGGCCTGTGTGTC & RTPCR \\
SDC2rev & GCATAAAACTCCTTAGTGGG & RTPCR \\
SDC3for & CGTGGCTGACGTAAGGACC & RTPCR \\
SDC3rev & CTCTAGTATGCTCTTCTGAA & RTPCR \\
SDC4for & CGAGAGACAGAGGTCATCGAC & RTPCR \\
SDC4rev & TGCGTAGAACTCATTGGTGG & RTPCR \\
bactfor & ATGGATGACGATATCGCTGCG & RTPCR \\
bactrev & CTAGAAGCATTTGCGGTGCAC & RTPCR \\
SDC2ecoRIfor & TATATAGAATTCGTAGGAGCCACATCCCTG & SDC2 cloning \\
SDC2ecoRIrev & TATATAGAATTCTTATGCATAAAACTCCTTAGTGG & SDC2 cloning \\
SDC4ecoRIfor & TATATAGAATTCGACTGGTTTGCGCTGTTGAA & SDC4 cloning \\
SDC4ecoRIrev & TATATAGAATTCTCATGCGTAGAACTCATTGGT & SDC4 cloning \\
IRESdelfor & GATATCCGCTAGCGCTACCGGACT & Deletion of CMV promoter from pIRES2-EGFP \\
IRESdelrev & ATGCATGGCGGTAATACGGT & Deletion of CMV promoter from PIRES2-EGFP \\
GFPfor & TATATAAAGCTTGCCACCATGGTGAGCAAG & GFP cloning \\
GFPrev & TATATAGGATCCATGATCTAGAGTCGCGGCC & GFP cloning \\
\hline
\end{tabular}


The GFP cDNA from pCAGGS was removed by EcoRI digestion, the product was gel purified then ligated to the EcoRI site of pCAGIPuro to create pCAGIPuro-GFP.

To insert the neomycin resistance cassette into the pCAGGS vector, pMC1neo polyA was digested with SalI and XhoI to release the neomycin resistance cassette, which was then ligated into the SalI site of pCAGGS to create pCAGGSneo-GFP.

For control purposes, the GFP cDNA was removed from pCAGGS by restriction digest with the enzyme EcoRI to create pCAGGS-ev. The neomycin resistance cassette from pMC1neo PolyA was then ligated to the SalI site of pCAGGS-ev to create pCAGGSneo-EV.

To create pIRES2-EGFP( $\beta$-actin), the EcoRI/SalI fragment of pLK444 [24] containing the $\beta$-actin promoter was gel purified and the overhangs were filled using Pfu polymerase. To remove the CMV promoter from pIRES2-EGFP (Clontech), PCR was performed using the primer pair IRESdelfor and IRESdelrev (Table 2). The primers were designed to yield a plasmid backbone of the pIRES2-EGFP containing an EcoRV site in place of the CMV promoter (pIRES2-EGFP-CMV). pIRES-EGFP-CMV was then cut with EcoRV, dephosphorylated and ligated to the bluntended $\beta$-actin fragment to generate $\operatorname{pIRES} 2-\operatorname{EGFP}(\beta-$ actin).

To create pLK444-GFP, the EGFP cDNA was amplified by PCR from pEGFP-N1 using the primer pair GFPfor and GFPrev (Table 2). The PCR product was digested with HindIII and BamHI and ligated to the HindIII/BamHI sites of pLK444 to yield pLK444-GFP.

\section{Transfection}

For transfection in suspension, 150,000 CCE cells were plated per well of a six-well plate $24 \mathrm{~h}$ prior to transfection. Cells were trypsinised and washed in serum free DMEM. $1 \mu \mathrm{g}$ DNA, $10 \mu \mathrm{l}$ lipofectamine (Invitrogen) and serum free DMEM to a final volume of $50 \mu \mathrm{l}$ were added to the cell pellet and incubated for $10 \mathrm{~min}$ at room temperature before transfer to a well of a 6-well plate containing $3 \mathrm{ml}$ culture medium (supplemented with FCS). Fresh medium was added after $24 \mathrm{~h}$. Fibroblasts were transfected with lipofectamine following standard procedures.

\section{Abbreviations}

BSA, bovine serum albumin; CMV, cytomegalovirus; EDTA, ethylenediaminetetraacetic acid; FACS, fluorescence-activated cell sorting;GFP, green fluorescent protein; IRES, internal ribosomal entry site; LIF, leukemia inhibitory factor; PBS, phosphate buffered saline; PDGFR, platelet derived growth factor receptor; SSEA, stage spe- cific embryonic antigen;VEGFA, vascular endothelial growth factor.

\section{Competing interests}

The author(s) declare that they have no competing interests.

\section{Authors' contributions}

ANA performed all of the experimental work presented in this paper. JRC and JRW both conceived of the study and were involved in drafting the manuscript. All of the authors have read and approved the final version of this manuscript.

\section{Acknowledgements}

This work was supported by the Wellcome Trust Program Grant 065940 to JRC and Wellcome Trust Studentship 069369 to ANA. We gratefully acknowledge the generous gift of the PCAGIPuro vector from Dr. Hitoshi Niwa of the RIKEN Centre for Developmental Biology, Kobe, Japan. In addition we would like to thank Dr. Clare Huxley of Imperial College for her hep and advice.

\section{References}

I. Wobus AM, Boheler KR: Embryonic stem cells: prospects for developmental biology and cell therapy. Physiol Rev 2005, 85:635-678.

2. Feraud O, Cao Y, Vittet D: Embryonic stem cell-derived embryoid bodies development in collagen gels recapitulates sprouting angiogenesis. Lab Invest 200I, 8I: I669-I68I.

3. Yamashita J, Itoh H, Hirashima M, Ogawa M, Nishikawa S, Yurugi T, Naito M, Nakao K, Nishikawa SI: FIk I-positive cells derived from embryonic stem cells serve as vascular progenitors. Nature 408:92-96.

4. Fathi F, Tiraihi T, Mowla SJ, Movahedin M: Transfection of CCE mouse embryonic stem cells with EGFP and BDNF genes by the electroporation method. Rejuvenation Res 2006, I:26-30.

5. Lakshmipathy U, Pelacho B, Sudo K, Linehan JL, Coucouvanis E, Kaufman DS, Verfaillie CM: Efficient transfection of embryonic and adult stem cells. Stem Cells 2004, 22:53I-543.

6. Ma H, Liu Q, Diamond SL, Perce EA: Mouse embryonic stem cells efficiently lipofected with nuclear localisation peptide result in a high yield of chimeric mice and retain germline transmission potency. Methods 2004, 33: I I3-120.

7. Chung S, Andersson T, Sonntag KC, Björklund L, Isacson O, Kim KS: Analysis of different promoter systems for efficient transgene expression in mouse embryonic stem cell lines. Stem Cells 2002, 20:139-145.

8. Lorenz P, Harnack U, Morgenstern R: Efficient gene transfer into murine embryonic stem cells by nucleofection. Biotechnol Lett 2004, 26: I589-I592.

9. Ward CM, Stern PL: The human cytomegalovirus immediateearly promoter is transcriptionally active in undifferentiated mouse embryonic stem cells. Stem cells 2002, 20:472-475.

10. Caron L, Bost M, Prot M, Hofman P, Binétruy B: A new role for the oncogenic high-mobility group $A 2$ transcription factor in myogenisis of embroyonic stem cells. Oncogene 2005, 24:628I-629l.

II. Chambers I, Colby D, Robertson M, Nichols J, Lee S, Tweedie S, Smith A: Functional expression cloning of Nanog, a pluripotency sustaining factor in embryonic stem cells. Cell 2003, I 1 3:643-55.

12. Suzuki H, Watabe T, Kato M, Miyazawa K, Miyazono K: Roles of vascular endothelial growth factor receptor 3 signaling in differentiation of mouse embryonic stem cell-derived vascular progenitor cells into endothelial cells. Blood 2005, I 05:2372-2379.

13. Fraser ST, Yamashita J, Jakt LM, Okada M, Ogawa M, Nishikawa S, Nishikawa S: In vitro differentiation of mouse embryonic stem 
cells: hematopoietic and vascular cell types. Methods Enzymol 2003, 365:59-72.

14. Cui L, Johkura K, Yue F, Ogiwara N, Okouchi Y, Asanuma K, Sasaki $\mathrm{K}$ : Spatial distribution and initial changes of SSEA-I and other cell adhesion-related molecules on mouse embryonic stem cells before and during differentiation. J Histochem Cytochem 2004, 52: | 447- I 457.

15. Nishikawa SI, Nishikawa S, Hirashima M, Matsuyoshi N, Kodama H: Progressive lineage analysis by cell sorting and culture identifies FlkI+VE-cadherin+ cells at a diverging point of endothelial and hematopoietic lineages. Development 1998, 125: I747-I757.

16. Redick SD, Bautch VL: Developmental platelet endothelial cell adhesion molecule expression suggests multiple roles for a vascular adhesion molecule. Am J Pathol 1999, I54: | |37-| | 47.

17. Niwa H, Yamamura KI, Miyazaki Jl: Efficient selection for high expression transfectants with a novel eukaryotic vector. Gene 1991, 108:193-200.

18. Alexopoulou AN, Multhaupt HA, Couchman JR: Syndecans in wound healing, inflammation and vascular biology. Int J Biochme Cell Biol 2007, 39:505-528.

19. Chen E, Hermanson S, Ekker SC: Syndecan-2 is essential for angiogenic sprouting during zebrafish development. Blood 2004, 103:1710-1719.

20. Cizmeci-Smith G, Langan E, Youkey J, Showalter LJ, Carey DJ: Syndecan-4 is a primary-response gene induced by basic fibroblast growth factor and arterial injury in vascular smooth muscle cells. Arterioscler Thromb Vasc Biol 1997, I7(I):172-180.

21. Echtermeyer F, Streit M, Wilcox-Adelman S, Saoncella S, Denhez F, Detmar M, Goetinck P: Delayed wound repair and impaired angiogenesis in mice lacking syndecan-4. J Clin Invest 200I, I07(2):R9-RI4.

22. Fears CY, Gladson CL, Woods A: Syndecan-2 is expressed in the microvasculature of gliomas and regulates angiogenic processes in microvascular endothelial cells. J Biol Chem 2006, 28I: I 4533-I 4536 .

23. Kojima T, Takagi A, Maeda M, Segawa T, Shimizu A, Yamamoto K, Matsushita T, Saito H: Plasma levels of syndecan-4 (ryudocan) are elevated in patients with acute myocardial infarction. Thromb Haemost 200I, 85:793-799.

24. Gunning P, Leavitt J, Muscat G, Ng SY, Kedes L: A human betaactin expression vector system directs high-level accumulation of antisense transcripts. Proc Natl Acad Sci USA 1987, 84:483I-48I5.
Publish with Bio Med Central and every scientist can read your work free of charge

"BioMed Central will be the most significant development for disseminating the results of biomedical research in our lifetime. "

Sir Paul Nurse, Cancer Research UK

Your research papers will be:

- available free of charge to the entire biomedical community

- peer reviewed and published immediately upon acceptance

- cited in PubMed and archived on PubMed Central

- yours - you keep the copyright 